\title{
Acute coronary artery occlusion and ischemia-related ventricular tachycardia during catheter ablation in the right ventricular outflow tract
}

Yosuke Nakatani ${ }^{1}$, Konstantinos Vlachos ${ }^{2}$, F. Daniel Ramirez ${ }^{3}$, Takashi Nakashima ${ }^{4}$, Pierre Jais ${ }^{5}$, Frederic Sacher ${ }^{6}$, and Romain Tixier ${ }^{5}$

${ }^{1}$ University of Toyama

${ }^{2}$ Evangelismos General Hospital of Athens

${ }^{3}$ Centre Hospitalier Universitaire de Bordeaux Hopital Cardiologique

${ }^{4} 1$. Electrophysiology and Ablation Unit and L'Institut de rythmologie et modélisation cardiaque (LIRYC)

${ }^{5}$ Centre Hospitalier Universitaire de Bordeaux

${ }^{6}$ Bordeaux University Hospital

September 11, 2020

\begin{abstract}
Coronary artery injury is a rare complication of catheter ablation in the right ventricular outflow tract (RVOT). Furthermore, acute myocardial ischemia usually causes polymorphic ventricular tachycardia (VT) or ventricular fibrillation. We herein describe a case in which catheter ablation for VT originating from the RVOT provoked ischemia-related VTs due to acute occlusion of the left anterior descending artery.
\end{abstract}

\section{Acute coronary artery occlusion and ischemia-related ventricular tachycardia during catheter} ablation in the right ventricular outflow tract

Yosuke Nakatani, MD; Konstantinos Vlachos, MD; F. Daniel Ramirez, MD; Takashi Nakashima, MD; Pierre Jais, MD; Frédéric Sacher, MD, PhD; Romain, Tixier, MD

Department of Cardiac Pacing and Electrophysiology, IHU Liryc, Electrophysiology and Heart Modeling Institute, Univ. Bordeaux, Bordeaux University Hospital (CHU), 33600 Pessac- Bordeaux, France

Funding: FDR is supported by a Banting Postdoctoral Fellowship from the Canadian Institutes of Health Research.

Disclosures: FS has received speaking honorarium from Biosense Webster, Abbott, Boston Scientific, Medtronic, Microport and Bayer Healthcare

Address for correspondence: Dr. Yosuke Nakatani, Department of Cardiac Pacing and Electrophysiology, Hôpital Cardiologique du Haut-Lévêque Avenue de Magellan, 33604, Bordeaux-Pessac, France. Telephone: +33-5-57656542; Fax: +33-5-57656509; E-mail: yosuke3gbst@gmail.com

\section{ABSTRACT}

Coronary artery injury is a rare complication of catheter ablation in the right ventricular outflow tract (RVOT). Furthermore, acute myocardial ischemia usually causes polymorphic ventricular tachycardia (VT) 
or ventricular fibrillation. We herein describe a case in which catheter ablation for VT originating from the RVOT provoked ischemia-related VTs due to acute occlusion of the left anterior descending artery.

\section{KEYWORDS}

Ventricular tachycardia; catheter ablation; right ventricular outflow tract; coronary artery; left anterior descending artery

\section{CASE PRESENTATION}

A 79-year-old man with dilated cardiomyopathy was hospitalized for the treatment of ventricular tachycardia (VT) with syncope. Echocardiography revealed diffuse mild hypokinesis of the left ventricle (LV) with an ejection fraction of $45 \%$. Cardiac magnetic resonance imaging identified non-specific fibrosis in the LV posterior wall, but no right ventricular abnormality was apparent. Coronary angiography showed no significant coronary artery stenosis (Fig. 1A). Radiofrequency catheter ablation (RFCA) was performed with intravenous midazolam, morphine, and acetaminophen. The baseline surface electrocardiogram showed atrial fibrillation with complete right bundle branch block and left axis deviation (Fig. 2A) as well as frequent runs of non-sustained VT (VT 1) (Fig. 3A). The morphology of VT 1 was a left bundle branch block pattern with a precordial transition in lead V4 and inferior axis. The activation map of VT 1 showed a centrifugal pattern from the septal aspect of the right ventricular outflow tract (RVOT), which was targeted with RFCA (irrigated tip catheter, maximum power $35 \mathrm{~W}$ ) (Fig. 1B). Maximum contact force exceeded $50 \mathrm{~g}$ at some RFCA sites. VT 1 was suppressed after 180 seconds of RFCA; however, other VTs (VT 2 and VT 3 ) occurred thereafter (Fig. 3B and 3C). The morphologies of VT 2 and VT 3 were similar to that of VT 1, but the precordial transition occurred in lead V3. The initiations of these VTs were accompanied by an acceleration in heart rate, and no preceding ventricular premature beats were observed. Additional RFCA was performed in the RVOT, targeting the earliest local ventricular potentials during the VTs. However, the frequency and duration of VTs only increased. At this point, ST-elevation in the precordial leads and in aVL was noted (Fig. 2B). A review of surface electrocardiograms revealed that ST-elevation appeared 4 minutes after RFCA for VT1, but the patient had remained asymptomatic. Coronary angiography confirmed a total occlusion of the mid left anterior descending artery (LAD) (Fig. 1C), which was treated with emergency coronary angioplasty. Following reperfusion, all VTs completely resolved. Merging three-dimensional computed tomography images with electroanatomical maps revealed that the RFCA sites were markedly close to the mid LAD (Fig. 1C). No VT recurrence was observed during six months' follow-up.

\section{DISCUSSION}

Coronary artery injury is a rare but important complication of RFCA in the RVOT.[1,2] Given the anatomical proximity of coronary arteries to the RVOT,[3] high-power ablation in this structure should be avoided as should excessive contact force. The use of irrigated-tip catheters may additionally increase the risk of coronary artery injury as it favors deeper lesion creation. Indeed, irrigated-tip catheters have been implicated in reported cases of LAD injury from RVOT ablation. [1,2]

There were several reasons for the delayed recognition of ST-elevation in this case. Even though the procedure was performed under conscious sedation, the patient was asymptomatic. Careful monitoring of the STsegment is particularly important in cases using general anesthesia as patient feedback is impossible, although as this example illustrates, caution is warranted in all cases. An awareness of the risk of coronary artery injury during RVOT ablation can be crucial as ST-segment changes are usually not the focus of attention during relevant procedures and can be subtle depending on which leads are being monitored in real-time. In this case, the attention paid to distinguishing between multiple morphologically similar VTs likely distracted us from changes in the ST-segment. Furthermore, the ST-segment changes were relatively minor in leads I, III, V1, and V6, which were the leads displayed alongside intracardiac electrograms (Fig. 2B).

That acute LAD occlusion resulted in monomorphic VTs is unusual; however, their occurrence only after the onset of ST-elevation and full suppression with cardiac reperfusion strongly argue for acute ischemia as the cause. Furthermore, the morphologies of VT 2 and VT 3 suggest LV exits close to the ischemic territory, 
with the QRS morphologies of VT 2 and VT 3 similar to that of VT 1 but with earlier precordial transitions. Traditionally, acute ischemia causes polymorphic VT or ventricular fibrillation, whereas monomorphic VT is typically associated with myocardial scar and reentry. In the present case, however, there was no evidence of scar in the expected culprit region and both VT 2 and VT 3 were preceded by heart rate accelerations and a lack of ventricular ectopics, which may suggest a non-reentrant mechanism. Although speculative, it is possible that acute ischemia increased automaticity or triggered activity by established mechanisms, such as increases in intracellular calcium, the production of free fatty acids and oxygen free radicals, acidosis, and increased catecholamine levels.[4]

To the best of our knowledge, this is the first reported case of ischemia-related VTs attributable to acute LAD occlusion from endocardial RVOT ablation. An awareness of the risk of acute coronary artery injury and resultant ischemia-related VTs during RVOT ablation could help avoid exacerbating this complication with further RFCA, particularly as the VT morphology changes can be subtle as was seen in the present case.

\section{REFERENCES}

1. Dilling-Boer D, Vanduynhoven P. Lessons learned from asymptomatic acute coronary occlusion complicating radiofrequency ablation of right ventricular outflow tract tachycardia. J Cardiovasc Electrophysiol 2015;26:1269-1272.

2. Benhayon D, Nof E, Chik WW, Marchlinski F. Catheter ablation in the right ventricular outflow tract associated with occlusion of left anterior descending coronary artery. J Cardiovasc Electrophysiol 2017;28:347-350.

3. Vaseghi M, Cesario DA, Mahajan A, Wiener I, Boyle NG, Fishbein MC, Horowitz BN, Shivkumar K. Catheter ablation of right ventricular outflow tract tachycardia: value of defining coronary anatomy $\mathrm{J}$ Cardiovasc Electrophysiol. 2006;17:632-637.

4. Mehta D, Curwin J, Gomes JA, Fuster V. Sudden death in coronary artery disease: acute ischemia versus myocardial substrate. Circulation 1997;96:3215-3223.

\section{FIGURES}

Figure 1
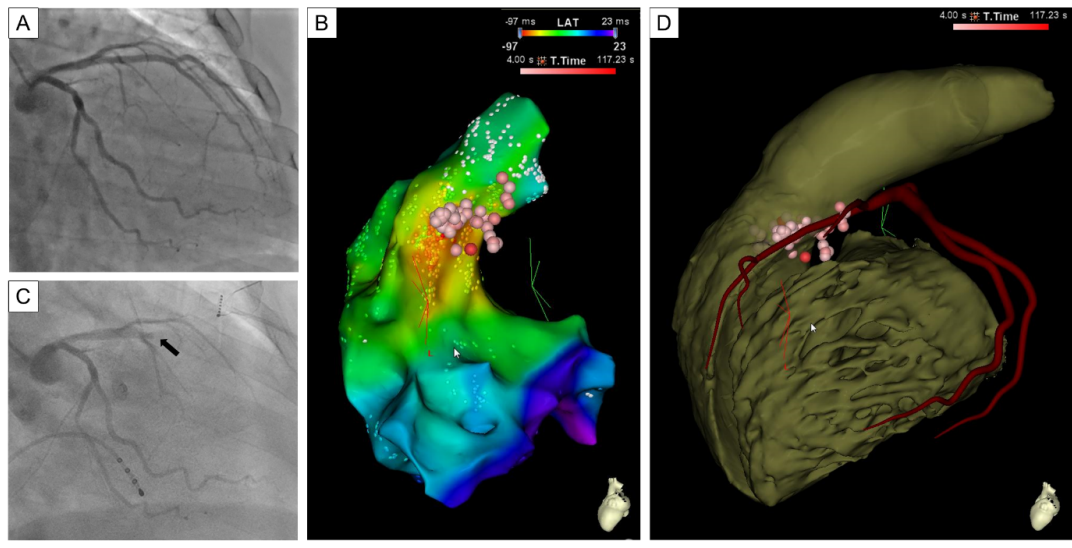

Figure 1. (A) Coronary angiography before ablation. (B) Activation map of the clinical ventricular tachycardia (VT 1). Tags on the map denote ablation sites. (C) Coronary angiography after ablation demonstrating an occluded left anterior descending artery (LAD) (arrow). (D) Three-dimensional cardiac computed tomography. Coronary arteries are shown in red. Tags on the map denote ablation sites, which are the same as in panel B. Note the proximity of ablation sites to the LAD. 
Figure 2

A. Baseline

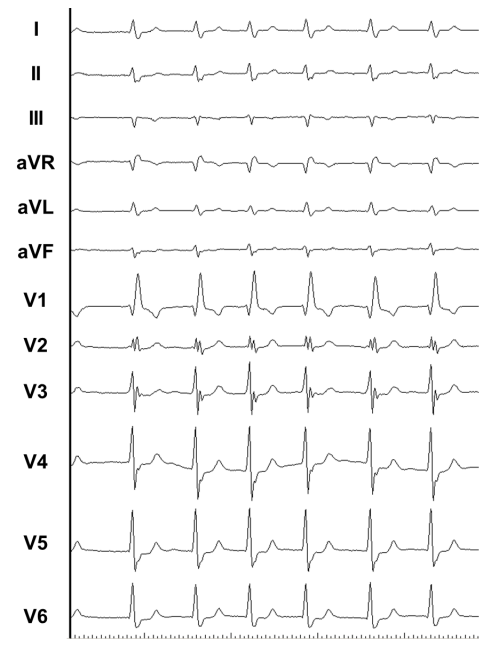

B. ST elevation

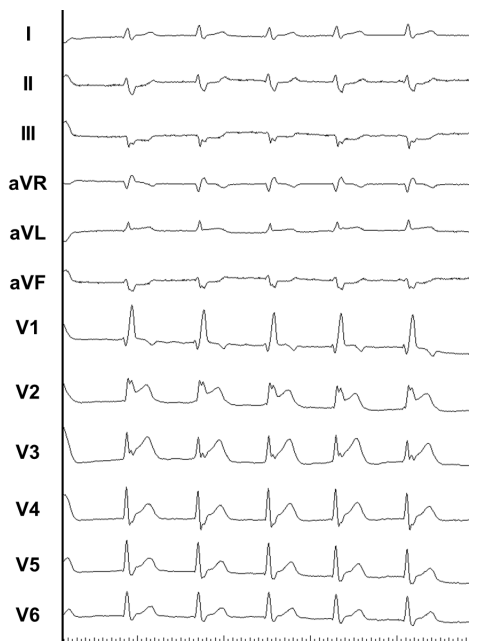

Figure 2. (A) Baseline surface electrocardiogram (ECG). (B) Surface ECG recorded during the procedure. Note new ST-elevation in the precordial leads, I, and aVL with reciprocal ST-depression in the inferior leads.

Figure 3

A. VT 1

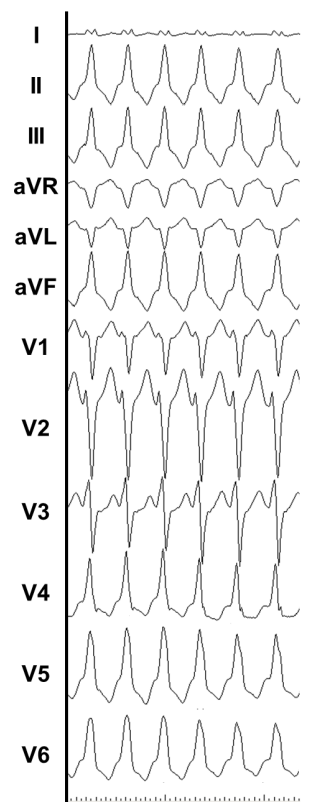

B. VT 2

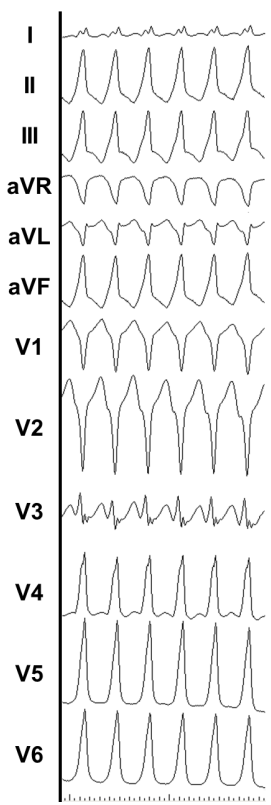

C. VT3

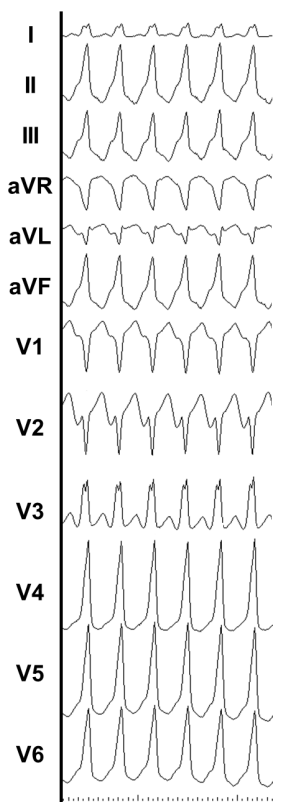

Figure 3. Surface electrograms of ventricular tachycardias (VTs). VT 1 (A), VT 2 (B), and VT 3 (C) were observed during the procedure. 